\title{
Pharmacology, Part 3B: Less Commonly Used Interventional Medications and Adjunctive Medications in General Nuclear Medicine
}

\author{
Geoffrey M. Currie \\ Faculty of Science, Charles Sturt University, Wagga Wagga, Australia, and Regis University, Boston, Massachusetts
}

CE credit: For CE credit, you can access the test for this article, as well as additional JNMT CE tests, online at https://www.snmmilearningcenter.org. Complete the test online no later than March 2022. Your online test will be scored immediately. You may make 3 attempts to pass the test and must answer $80 \%$ of the questions correctly to receive $1.0 \mathrm{CEH}$ (Continuing Education Hour) credit. SNMMI members will have their CEH credit added to their VOICE transcript automatically; nonmembers will be able to print out a CE certificate upon successfully completing the test. The online test is free to SNMMI members; nonmembers must pay $\$ 15.00$ by credit card when logging onto the website to take the test.

\section{A companion article to Part 3B, "Pharmacology, Part 3A," appeared in the December issue of JNMT, page 326.}

The understanding of key principles in pharmacology is essential to the clinical and research nuclear medicine practitioner. The scope of practice of the nuclear medicine technologist requires understanding of the indications, contraindications, warnings, precautions, proper use, drug interactions, and adverse reactions for interventional and adjunctive medications. This article is the companion to the third in a series of articles that aims to enhance the understanding of pharmacologic principles relevant to nuclear medicine. This article will build on the introductory concepts, terminology, and principles of pharmacology explored in previous articles in the series. Specifically, this article will focus on the pharmacologic principles associated with less commonly used interventional medications and adjunctive medications encountered in general nuclear medicine practice. Future articles will address the pharmacology related to nuclear cardiology, the emergency crash cart, and contrast media associated with CT and MRI.

Key Words: adjunctive; intervention; medication; pharmacology

J Nucl Med Technol 2019; 47:3-12

DOI: $10.2967 /$ jnmt.118.215053

\section{A} s outlined in the companion article (1), the scope of practice for nuclear medicine technologists (2) requires that they possess a thorough understanding and knowledge of indications, contraindications, warnings, precautions, proper use, drug interactions, and adverse reactions for

\footnotetext{
Received May 23, 2018; revision accepted Aug. 2, 2018.

For correspondence or reprints contact: Geoffrey Currie, Faculty of Science, Locked Bag 588, Charles Sturt University, Wagga Wagga 2678, Australia.

E-mail: gcurrie@csu.edu.au

Published online Aug. 23, 2018.

COPYRIGHT @ 2019 by the Society of Nuclear Medicine and Molecular Imaging
}

each medication to be used. This scope demands sound understanding of the principles of pharmacology provided in earlier articles in this series $(3,4)$. The content presented in these previous articles should be considered assumed knowledge, and those foundation principles will not be redefined here. The list of medications used either interventionally or adjunctively is long, and an exhaustive examination of all medications used in nuclear medicine is beyond the scope of this paper. Those common medications that are used adjunctively or are less common interventionally in general nuclear medicine will be outlined in detail. These have been summarized in Tables 1 and 2 .

\section{LESS COMMON INTERVENTIONAL STUDIES}

There are several less frequently performed interventional procedures in general nuclear medicine. Acetazolamide evaluation of cerebral flow reserve and the use of $\mathrm{H}_{2}$ histamine antagonists or proton pump inhibitors to enhance detection of Meckel diverticulum are discussed below and summarized in Table 1. Glucagon and pentagastrin have been excluded, as these previously used approaches in Meckel diverticulum detection have been largely replaced by $\mathrm{H}_{2}$ histamine and proton pump inhibitors. Rarely used interventions for diabetic gastroparesis assessment have also been excluded (metoclopramide and erythromycin).

\section{Acetazolamide (Diamox; Wyeth Holdings Corp.)}

General Information/Drug Class. Acetazolamide is a sulfonamide carbonic anhydrase inhibitor (5-8). Regional cerebral blood flow and cerebral flow reserve can be assessed by perfusion imaging at rest and after vasodilation $(8,9)$. Decreased flow reserve will not demonstrate the same increased flow in response to vasodilation as areas with a normal vascular supply $(8,9)$.

Mode of Action. The predominant site for carbonic anhydrase is in the proximal tubule of the kidney (Fig. 1 in the companion article [1]), where it facilitates the carbon dioxide 


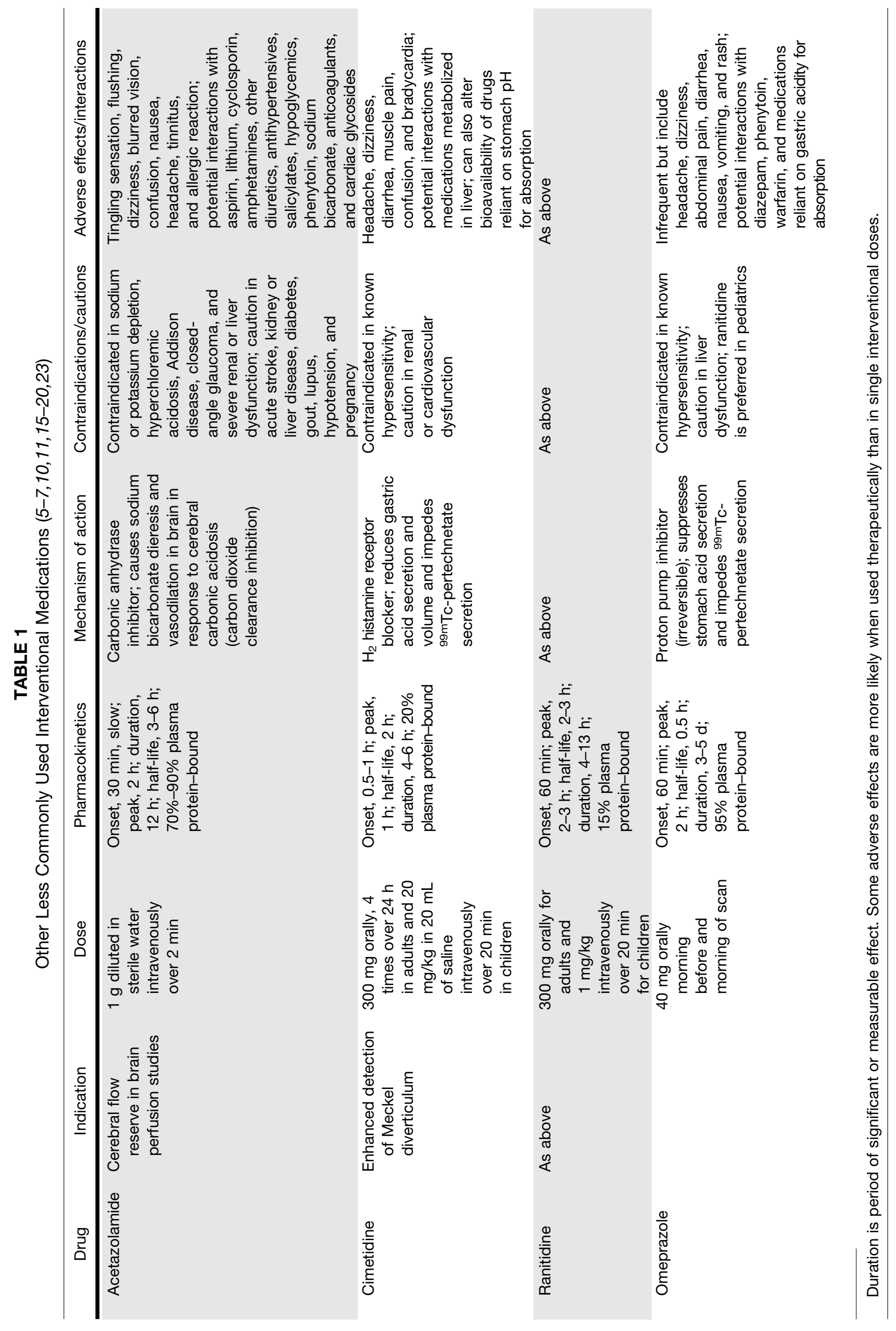

4 Journal of Nuclear Medicine Technology • Vol. 47 • No. 1 • March 2019 


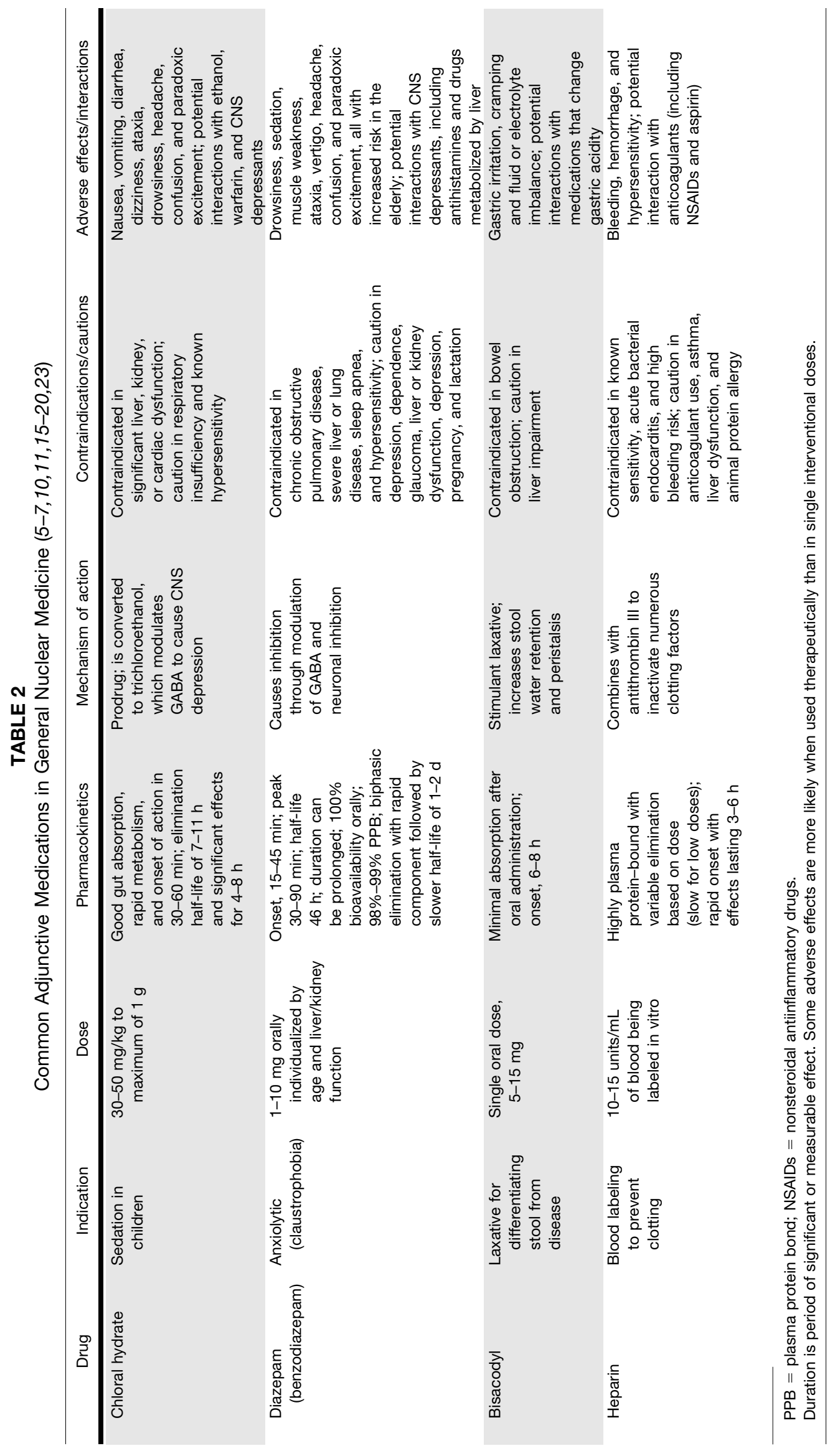


hydration/carbonic acid dehydration reaction $(5,6,10,11)$. It is this reaction that controls bicarbonate reabsorption in the kidney. By inhibiting carbonic anhydrase, acetazolamide effectively causes sodium bicarbonate diuresis and an overall reduction of total-body stores of bicarbonate $(6,10)$. Urinary alkalinization is linked to metabolic acidosis, and this results from the profound inhibition and blockade of bicarbonate reabsorption in the proximal tubule $(5,6)$. Rapid and potent cerebral vasodilation follow acetazolamide injection because of the decrease in $\mathrm{pH}$ resulting from cerebral carbonic acidosis after inhibition of carbon dioxide clearance $(12,13)$. The increased cerebral perfusion is due to intra- and extracellular acidosis (Fig. 1) (14). Cerebral flow response is not immediate, with a slow increase over a period of 15-25 min before returning to normal (15). This effect increases cerebral blood volume by $9 \%$ and cerebral blood flow by $50 \%$ in normal vascular territories $(1,15,16)$. Although normal vessels readily dilate, diseased vessels do not, allowing the interventional study (compared with baseline) to exaggerate the blood flow difference between regions with normal and diseased vasculature $(8,9,17)$.

Pharmacokinetics. Acetazolamide has an intravenous bioavailability of $100 \%(16,18)$. It has $70 \%-90 \%$ plasma protein binding, and $90 \%$ is excreted unchanged in urine $(16,18)$. The elimination half-life is $3-6 \mathrm{~h}(8,9,16,18)$. After intravenous administration, onset of action occurs within $30 \mathrm{~min}$, with peak activity seen by $2 \mathrm{~h}$ and a duration of effect of $12 \mathrm{~h}$ from a single dose $(6,8,9,16-18)$.

Usual Indications. There are several other indications documented in the literature including use as a weak diuretic $(6,11,16,17,19)$; in the acute management of glaucoma

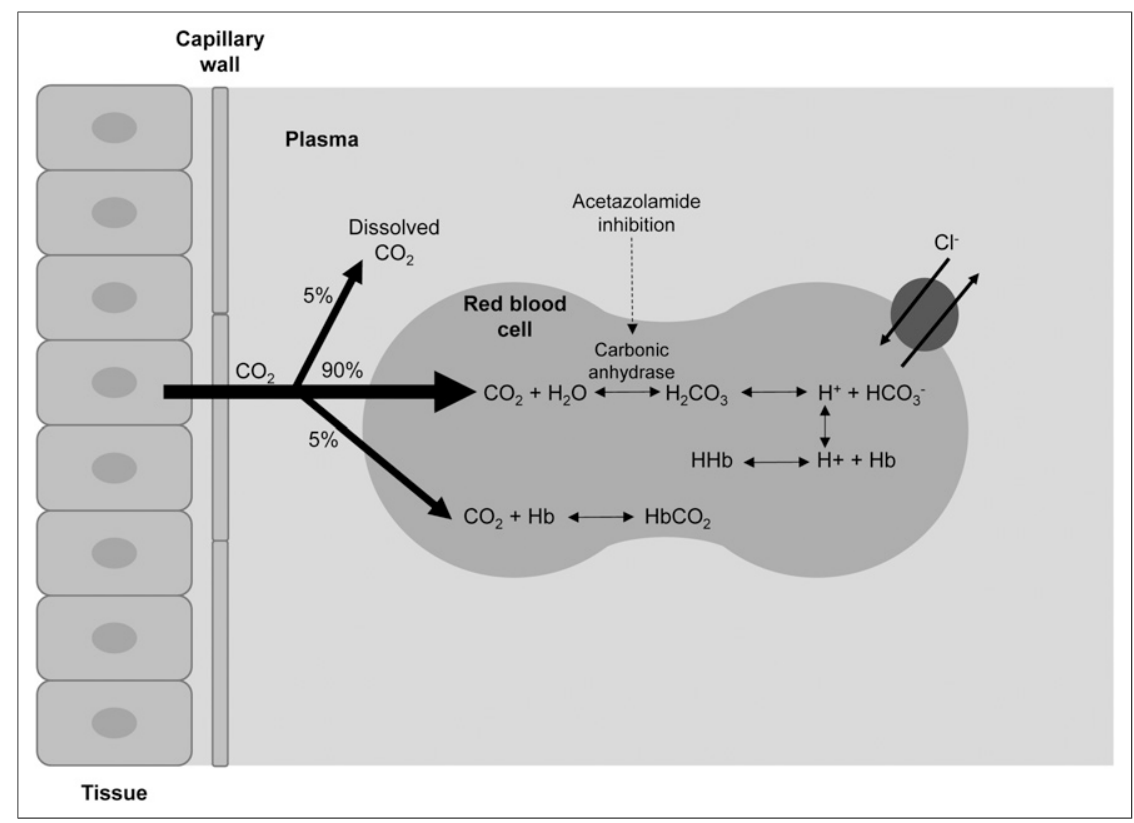

FIGURE 1. Schematic representation of impact of acetazolamide in inhibiting carbonic anhydrase and increasing serum carbon dioxide, which leads to increased cerebral perfusion. by minimizing the intraocular pressure $(6,11,16,19,20)$; as second- or third-line therapy in epilepsy, particularly in absence seizures, tonic-clonic seizures, and myoclonic seizures $(16,19)$; as a preferred agent for prophylaxis of altitude sickness syndrome $(11,16,19)$; for the treatment of macular edema, Meniere's disease, and neuromuscular disorders and to decrease intracranial pressure in idiopathic intracranial hypertension (16); and to decrease nephrotoxicity in patients taking methotrexate (16).

Use in Nuclear Medicine. Acetazolamide is used to increase cerebral blood flow for performing a stress test on the brain; for assessing cerebral flow reserve in patients with transient ischemic attacks, stroke, carotid artery stenosis, or other vascular disorders; and for differentiating vascular dementia from other causes of dementia $(8,9,15,17)$.

Proper Use and Dose Administration. The standard dose is $1 \mathrm{~g}$ of acetazolamide diluted in $10 \mathrm{~mL}$ of sterile water, which is then administered by slow intravenous infusion (over $2 \mathrm{~min}$ ) $(8,9,17)$. Typically, this administration is done $25-30 \mathrm{~min}$ before the radiopharmaceutical administration $(7,8)$.

Contraindications. Acetazolamide is contraindicated in cases of sodium or potassium depletion, hyperchloremic acidosis, Addison disease, adrenal insufficiency, and marked hepatic or renal impairment $(16,19)$. It is also contraindicated in hepatic cirrhosis because of risk of hepatic encephalopathy $(7,17)$. It should not be used in patients who have chronic closed-angle glaucoma (16).

Warnings and Precautions. Caution should be applied with acetazolamide use in known kidney or liver disease, diabetes, gout, lupus, hypotension, and pregnancy (16). Care should be taken when there is a known sulfonamide allergy $(16,17)$. Acetazolamide can cause hyperglycemia in diabetic patients $(16,17)$. Severe electrolyte imbalance can occur because of diuresis, severe renal disease may lead to nephrotoxicity, and caution should be exercised in patients with increased cerebral pressure (17). Acetazolamide is excreted in breast milk but need not be ceased even for therapeutic doses (as opposed to single interventional administrations) (16).

Adverse Reactions. For acetazolamide, any adverse reactions tend to be mild and transient but can include tingling sensations (extremities and mouth particularly), blurred vision, headache, dizziness, and confusion $(5,7,8,17)$. Some patients experience flushing, nausea, tinnitus, and changes in taste (17). The diuretic effect can lead to urinary urgency (17). These adverse reactions can be minimized using the slow intravenous infusion (over $2 \mathrm{~min}$ ). Longer-term use (therapeutic doses) can also lead to sun 
sensitivity, loss of appetite, increased body hair, hearing loss, tachycardia/arrhythmia, bleeding/bruising/hematuria, and mood changes (16).

Common Interactions. Acetazolamide has several important interactions that need consideration. First, it makes the urine alkaline and therefore decreases excretion of urine (16), potentially enhancing the effects of renally excreted or metabolized drugs (e.g., amphetamines and ciclosporin). Second, if used in conjunction with aspirin and other salicylates, acetazolamide can cause acidosis and central nervous system (CNS) toxicity (16). Third, if the patient is taking other antiepileptic drugs, the effects can be cumulative (16). Finally, alkalinization of the urine increases the solubility of methotrexate in the urine (16). Consequently, excretion of methotrexate is enhanced, and this effect can be used to decrease nephrotoxicity in those patients (16). There are several other reported interactions between acetazolamide and other medications potentially causing toxicity, including, but not limited to, carbamazepine, lithium, mexiletine, methadone, phenobarbital, phenytoin, and quinidine (21).

\section{Cimetidine (Tagamet; GlaxoSmithKline)}

General Information/Drug Class. The gastric mucosa concentrates ${ }^{99 \mathrm{~m}} \mathrm{Tc}$-pertechnetate, and this includes in Meckel diverticula (17). Cimetidine is an $\mathrm{H}_{2}$ histamine receptor antagonist that reduces gastric acidity $(5,10,15,17,18,20)$. The detection of a Meckel diverticulum can be enhanced by either increasing the uptake at the site or by reducing migration of activity away from the site (15). Although the ${ }^{99 m}$ Tc-pertechnetate accumulation is not affected, the change in gastric acidity in response to cimetidine reduces the secretions into the gastric lumen, concentrating the radiopharmaceutical $(7,17,20)$. This effect also applies to Meckel diverticula, enhancing visualization (17).

Mode of Action. Histamine is released from mast cells and stimulates $\mathrm{H}_{2}$ receptors in the gastric parietal cells to increase gastric acid secretion $(5,6,10,18)$. Cimetidine is an $\mathrm{H}_{2}$ histamine antagonist that inhibits histamine stimulation of receptors (Fig. 2) and, thus, reduces both the volume and the concentration of hydrochloric acid in the stomach by as much as $50 \%-70 \%(5-7,10,17,18,20,22)$.

Pharmacokinetics. Cimetidine has an oral bioavailability of $60 \%-70 \%(16,18)$. It has $20 \%$ plasma protein binding and is partially (25\%) metabolized in the liver, with $50 \%$ of the oral dose excreted unchanged in urine $(16,18)$. The elimination half-life is $2 \mathrm{~h}(16,18)$, but this can be increased in renal dysfunction (16). After oral administration, onset of action occurs within 30-60 min, with peak activity seen at $60 \mathrm{~min}$ if taken on an empty stomach and $120 \mathrm{~min}$ with food, and significant effects last 4-6 h (16-18). Although cimetidine crosses the placenta and is excreted in breast milk, it is safe to use in both situations (16).

Usual Indications. Cimetidine is used to treat peptic ulcer disease, gastroesophageal reflux, dyspepsia, and stress ulcers by reducing gastric acid secretion $(5,6,10,16,18)$. It has also been used for people at risk of aspiration during childbirth or general anesthesia (16).

Use in Nuclear Medicine. Cimetidine enhances radiopharmaceutical accumulation and visualization in Meckel diverticulum, improving the sensitivity of the test $(17,20)$.

Proper Use and Dose Administration. Cimetidine can be administered orally as an 800- to 1,600-mg single dose $24 \mathrm{~h}$ before the Meckel diverticulum study $(6,17)$. Alternatively, $300 \mathrm{mg}$ orally, 4 times during the $24 \mathrm{~h}$ before the study, can be used $(7,20)$. Oral administration is not always practical because of compliance issues associated with the regime, so a single intravenous dose of $300 \mathrm{mg}$ in $100 \mathrm{~mL}$ of $5 \%$ dextrose for adults over $20 \mathrm{~min}$ (or $20 \mathrm{mg} / \mathrm{kg}$ in children) $1 \mathrm{~h}$ before the study can be used $(7,17,20)$. The intravenous dose can be diluted in $20 \mathrm{~mL}$ of normal saline and administered over $2 \mathrm{~min}$, but the adverse reaction rate might increase (17).

Contraindications. Known hypersensitivity to the medication is a contraindication for use (7).

Warnings and Precautions. Renal impairment should be managed by a reduced dose, and slow intravenous administration should be used in preference to rapid bolus administration for patients with cardiovascular impairment (16).

Adverse Reactions. Cimetidine is well tolerated and has a good safety profile $(16,18)$. Nonetheless, patients may experience dizziness, confusion, headache, diarrhea, or bradycardia $(5,10,16-18)$. Adverse reactions tend to self-resolve but can be limited by extending the intravenous administration over a longer period (e.g., up to $20 \mathrm{~min}$ ) (17). Cimetidine has a weak antiandrogenic effect; gynecomastia, decreased libido, and impotence have been shown to occasionally occur in men, but these conditions are usually reversible (16).

Common Interactions. Cimetidine and other $\mathrm{H}_{2}$ antagonists can reduce the absorption of drugs reliant on gastric acidity, including dasatinib, ketoconazole, and itraconazole (16). Cimetidine decreases hepatic metabolism of warfarin, phenytoin, propranolol, nifedipine, chlordiazepoxide, diazepam, tricyclic antidepressants, lidocaine, theophylline, and metronidazole, thus increasing serum levels of these medications $(7,16,18)$.

\section{Ranitidine (Zantac; GlaxoSmithKline)}

General Information/Drug Class. Ranitidine is an $\mathrm{H}_{2}$ histamine antagonist that reduces gastric acidity in the same way as cimetidine, discussed above. Ranitidine and cimetidine share the same drug class, general information, mode of action (Fig. 2), clinical use, use in nuclear medicine, contraindications, and precautions. Adverse reactions and common interactions have similarities but also some important differences that should be highlighted.

Pharmacokinetics. Ranitidine has an oral bioavailability of $50 \%(16,18)$. It has $15 \%$ plasma protein binding and is partially $(4 \%-6 \%)$ metabolized in the liver, with $30 \%$ of the oral dose excreted unchanged in urine $(16,18)$. The elimination half-life is $2-3 \mathrm{~h}(16,18)$, but this can be increased in renal dysfunction (16). After oral administration, onset of action occurs within 60 min, with peak activity seen at 2-3 h (independent of fasting) and significant effects lasting 4-13 h (16-18). 


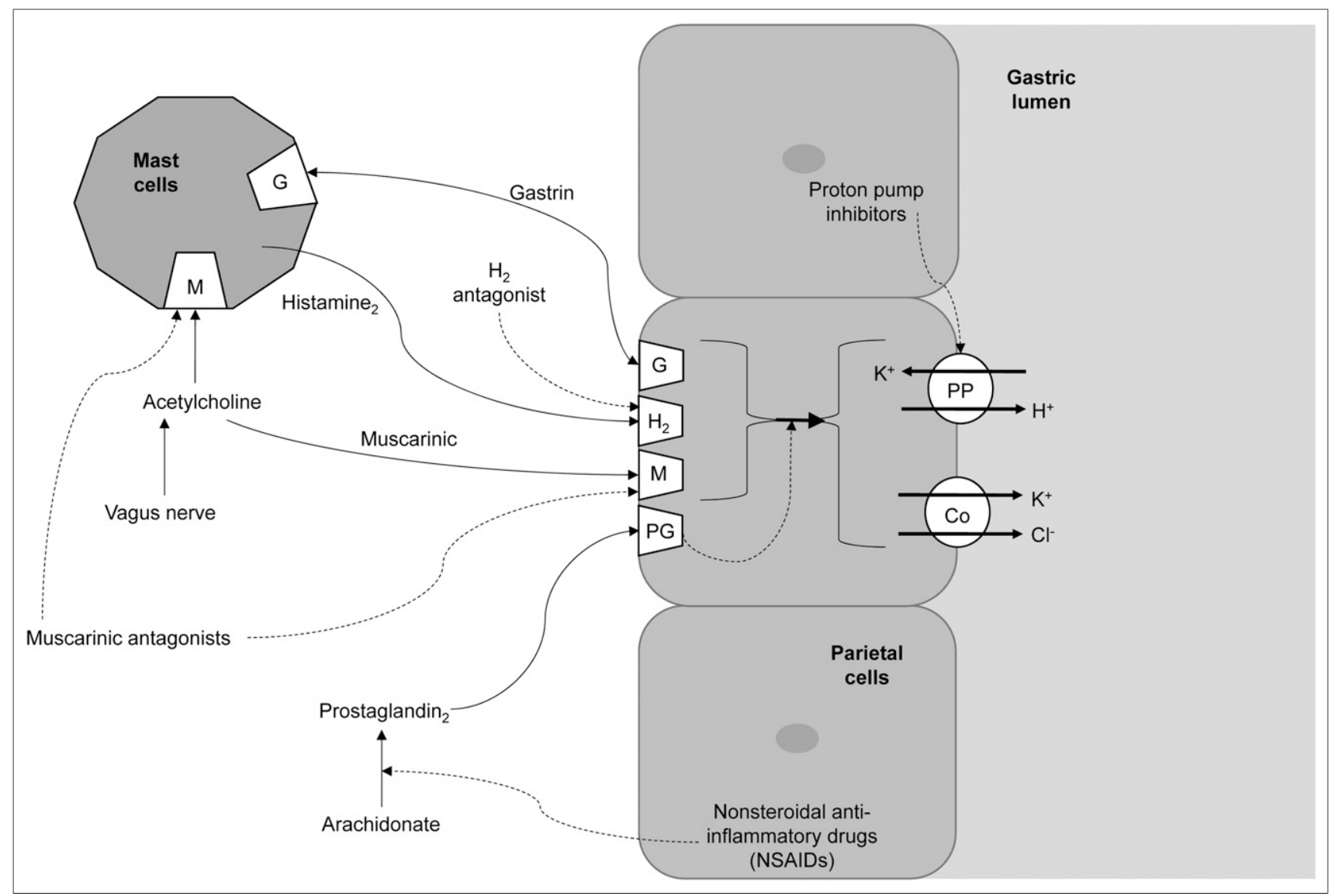

FIGURE 2. Schematic representation of action of receptors responsible for gastric acid secretion (solid lines) and inhibitors of gastric acid secretion (dashed lines). Co = cotransporter.

Ranitidine crosses the placenta and is excreted in breast milk but is safe to use in both pregnancy and breastfeeding (16).

Proper Use and Dose Administration. Ranitidine is administered orally as a 300-mg dose to adults or as $1 \mathrm{mg} / \mathrm{kg}$ to a maximum dose of $50 \mathrm{mg}$ intravenously over $20 \mathrm{~min}$ in children $(6,20)$. The imaging procedure commences $1 \mathrm{~h}$ after ranitidine administration (20).

Adverse Reactions. The adverse reactions are the same for ranitidine as for cimetidine, except ranitidine does not have the androgenic effects associated with cimetidine (16).

Common Interactions. Unlike cimetidine, ranitidine does not affect cytochrome P450 and, therefore, has little effect on the metabolism of other medications in the liver $(16,18)$. As with other $\mathrm{H}_{2}$ antagonists, ranitidine alters absorption of medications reliant on gastric acidity for absorption as outlined above for cimetidine (16).

\section{Omeprazole}

General Information/Drug Class. The detection of a Meckel diverticulum can be enhanced either by increasing the uptake at the site or by reducing migration of activity away from the site (15). Proton pump inhibitors decrease gastric acidity, increasing the availability of radiopharmaceutical for accumulation in the Meckel diverticulum (15).

Mode of Action. Omeprazole is a proton pump inhibitor that inhibits the secretion of gastric acid by inhibiting the proton pump $(5,6,10,16,18)$. The proton pump is the enzyme system of hydrogen/potassium adenosine triphosphatase in the gastric parietal cell (Fig. 2) $(5,6,10,16,18)$. In essence, when sufficient omeprazole accumulates or binds to the proton pump, the final step in acid production is inhibited, thus suppressing acid secretion $(5,6,10,18)$. The action is irreversible inhibition, which is reflected in the long duration of effect relative to the elimination half-life (18).

Pharmacokinetics. Omeprazole is rapidly but variably absorbed after oral administration, with an oral bioavailability of $30 \%-40 \%(16,18)$. It has $95 \%$ plasma protein binding and is extensively metabolized in the liver, with $80 \%$ of metabolites excreted in urine and the remaining $20 \%$ by feces $(16,18)$. The elimination half-life is $0.5 \mathrm{~h}$ $(16,18)$, but this can be increased in renal dysfunction (16). After oral administration, onset of action occurs within $60 \mathrm{~min}$, with peak activity seen at $120 \mathrm{~min}$ and significant effects lasting 3-5 d (16-18). Omeprazole has increased bioavailability in the elderly and those with liver dysfunction (16). 
Usual Indications. Omeprazole is used for the treatment of peptic ulcer, nonsteroidal antiinflammatory drug-induced ulceration, esophageal erosion due to acid reflux, and Zollinger-Ellison syndrome $(5,6,10,16,18)$. It is generally used in conditions for which gastric acid inhibition may relieve symptoms, including aspiration, dyspepsia, gastroesophageal reflux disease, and peptic ulcer (16). Proton pump inhibitors have also been used to reduce gastric acidity in the management of Helicobacter pylori (18).

Use in Nuclear Medicine. Omeprazole is used to enhance radiopharmaceutical accumulation in Meckel diverticulum by inhibiting the excretion from parietal cells (not affecting initial uptake).

Proper Use and Dose Administration. Omeprazole comes as a capsule formulation, which should be taken orally whole and not crushed, chewed, or opened (18). Therapeutically, omeprazole is administered as a single 20- to 40-mg dose daily for 4-8 wk $(6,18)$. For Meckel diverticulum preparation and in consideration of pharmacokinetic information, omeprazole should be administered as a 40$\mathrm{mg}$ dose on the morning preceding the scan and again on the day of the scan.

Contraindications. Omeprazole use is contraindicated in patients with known sensitivity (18).

Warnings and Precautions. The main precaution for omeprazole use is in patients with liver dysfunction $(16,18)$. There is a potential risk of drug accumulation (due to a high degree of liver metabolism) $(16,18)$; however, this risk is unlikely to present issues for isolated use as an interventional medication for Meckel diverticulum imaging.

Adverse Reactions. Omeprazole is well tolerated, and adverse reactions are minor (18). The risk of adverse reactions is further reduced with an isolated interventional dose. Nonetheless, the possibility of several adverse reactions should be considered, including abdominal pain, dizziness, headache, nausea, vomiting, diarrhea, flatulence, and rash $(5,16,18)$.

Common Interactions. Omeprazole is metabolized by cytochrome P2C19 and cytochrome P3A4 and so can alter the metabolism of many other medications $(16,18)$. Of note is the potential of omeprazole to increase plasma concentrations of diazepam, phenytoin, and warfarin $(16,18)$. Omeprazole can also decrease absorption of medications reliant on stomach acidity, including atazanavir, dasatinib, ketoconazole, and itraconazole $(16,18)$.

\section{COMMON ADJUNCTIVES IN GENERAL NUCLEAR MEDICINE}

There are several adjunctive medications used in general nuclear medicine, with the most common being sedatives, anxiolytics, laxatives, and anticoagulants. Specific medications used for each purpose will vary among clinical centers. Consequently, a prototype approach to medication selection will be adopted to allow a general understanding that can be translated for more specific application when alternative medications are used. For example, diazepam is the prototype anxiolytic medication and is used widely clinically and therapeutically; however, some sites may use alternative benzodiazepams (e.g., temazepam) or other drug classes (e.g., barbiturates). As outlined in Table 2, this discussion will focus on chloral hydrate as a sedative, diazepam as an anxiolytic, bisacodyl as a laxative, and heparin as an anticoagulant.

\section{Chloral Hydrate}

General Information/Drug Class. Chloral hydrate has a simple chemical structure that is similar to ethanol and chloroform $(6,18)$. A combination of chloral hydrate with alcohol produces the potentially toxic additive effects known as "knockout drops" or a "Mickey Finn" $(16,18)$.

Mode of Action. Chloral hydrate is a prodrug that is converted to trichloroethanol $(5,18)$. It is a powerful hypnotic with CNS depressant properties that has been used for pediatric sedation in nuclear medicine, but its mode of action remains uncertain $(5,18)$. It is thought to act by modulating the inhibitory properties of $\gamma$-aminobutyric acid (GABA) in neurotransmission (5).

Pharmacokinetics. Chloral hydrate is absorbed well from the gut and is rapidly metabolized to trichloroethanol in the liver (5). The elimination half-life is 7-11 h (16). After oral administration, onset of action (sedation) occurs within 30$60 \mathrm{~min}$, with significant effects lasting 4-8 h (16).

Usual Indications. Chloral hydrate is used as a sedative, a hypnotic, and a premedication for medical procedures $(5,6,16,18)$.

Use in Nuclear Medicine. In nuclear medicine, chloral hydrate is used for the sedation of pediatric patients for imaging procedures (18).

Proper Use and Dose Administration. The prescribed pediatric dose is $30-50 \mathrm{mg} / \mathrm{kg}$ to a maximum of $1 \mathrm{~g}$ as an oral liquid given 45-60 min before the procedure (16). Doses of $100 \mathrm{mg} / \mathrm{kg}$ up to $2 \mathrm{~g}$ can be used in children with respiratory monitoring (16).

Contraindications. Chloral hydrate is contraindicated in significant liver, kidney, or cardiac dysfunction (16).

Warnings and Precautions. Chloral hydrate should be used with caution in respiratory insufficiency (16). Hypersensitivity reactions can occur, as can paradoxic excitement. Because the effects can last more than $24 \mathrm{~h}$, driving or using machinery should be avoided (16). Chloral hydrate is excreted in breast milk and can cause infant sedation (16).

Adverse Reactions. There are several common adverse reactions to chloral hydrate, including nausea, vomiting, diarrhea, dizziness, ataxia, drowsiness, lightheadedness, headache, hallucination, confusion, and paradoxic excitement $(5,16)$. Hypersensitivity can lead to rash (16). Gastric irritation, abdominal distention, and flatulence have also been reported (16). It is possible with overdose to cause respiratory and cardiac depression $(5,18)$.

Common Interactions. Alcohol is the main interaction because trichloroethanol competes with ethanol for metabolism 
$(5,18)$. There is a potential additive effect associated with concurrent use of other CNS depressants $(16,18)$. Chloral hydrate is known to alter the effects of warfarin (16).

\section{Diazepam (Valium; Hoffmann-La Roche)}

General Information/Drug Class. Benzodiazepams are widely used hypnotics and anxiolytics because of their superior safety profile, which includes reduced dose-related effects, fatality from toxicity and overdose, abuse potential, adverse effects, and serious drug interactions $(5,6,18)$. Diazepam is the prototype diazepam, with newer variations having shorter durations of effect (e.g., lorazepam) and thus further improved safety profiles $(6,18)$.

Mode of Action. Diazepam is a CNS depressant that has specific functions to reduce anxiety $(5,6,10,18)$. Diazepam acts on the GABA receptor $(5,6,10,18)$. GABA is an endogenous inhibitory neurotransmitter that causes increased chloride permeability, hyperpolarization, and decreased excitability of the neuron $(5,6,10,18)$. Diazepam is not a GABA agonist but, rather, modulates the binding of GABA to GABA receptor A, further inhibiting the neuron $(5,10,18)$.

Pharmacokinetics. Diazepam is a long-acting benzodiazepam, with metabolites that have their own activity (e.g., temazepam) $(10,18)$. It has an oral bioavailability of almost $100 \%$, being easily and completely absorbed after oral administration (16). It has $98 \%-99 \%$ plasma protein binding and is extensively metabolized in the liver, with excretion in urine in the form of conjugated or free metabolites $(16,18)$. The elimination half-life is biphasic, with a rapid elimination phase followed by a second phase with a half-life of 1-2 d (16). After oral administration, onset of action occurs within 1545 min depending on the presence or absence of food, with peak activity seen at 30-90 min and significant effects being prolonged by the $2-5 \mathrm{~d}$ half-life of active metabolites (16). Diazepam crosses the placenta and is excreted in breast milk; it should therefore be used with caution in pregnancy and breastfeeding (16).

Usual Indications. Diazepam is used for short-term management of anxiety or muscle spasm, as a premedication for sedation, and as an adjunctive medication for managing seizures in the acute setting $(5,6,10,16,18)$.

Use in Nuclear Medicine. Diazepam is used to minimize anxiety in the claustrophobic patient, especially those undergoing SPECT, PET, and MRI.

Proper Use and Dose Administration. Diazepam can be administered intravenously, intramuscularly, rectally, and orally; however, for ease of use as an anxiolytic in nuclear medicine, the oral route is typically used. Doses should be individualized for age, liver and kidney function, and purpose, with typical doses ranging from 1 to $10 \mathrm{mg}(16,18)$. If diazepam is being applied for sedation in adults, $10-20 \mathrm{mg}$ intravenously over 2-4 min is used (16).

Contraindications. Diazepam is contraindicated in patients with chronic obstructive pulmonary disease, other severe respiratory diseases, severe liver disease, sleep apnea, glaucoma, myasthenia gravis, dependence on the drug, and hypersensitivity to the drug $(16,18)$. Diazepam use should be avoided in those who already have CNS depression $(16,18)$.

Warnings and Precautions. Diazepam should be used for only short periods, and withdrawal can be protracted. It should be used with caution in glaucoma, liver and kidney dysfunction, depression, psychosis, the elderly or very young, pregnancy, and breastfeeding $(16,18)$.

Adverse Reactions. Adverse reactions have an increased risk of occurrence in the elderly (6). The most common adverse reactions to diazepam are drowsiness, sedation, muscle weakness, and ataxia (16). Less frequently, patients may experience vertigo, headache, confusion, and, with ongoing use, depression, dysarthria, changes in libido, tremor, visual disturbances, urinary issues, gastrointestinal disturbances, and amnesia (16). Paradoxic excitement is possible $(16,18)$.

Common Interactions. Additive effects may be seen with other CNS depressants, including alcohol, sedating antihistamines, opioids, sedatives, and antidepressants $(16,18)$. Many drugs metabolized in the liver, including $\mathrm{H}_{2}$ antagonists, can inhibit diazepam metabolism and thus prolong its effect $(16,18)$.

\section{Bisacodyl}

General Information/Drug Class. There are several classifications for laxatives that might be used for interventional purposes $(5,6,10,18)$. Fecal softeners (e.g., docusate) use wetting agents to mix water and fatty substances with the feces but have a 1- to 3-d onset of action. Bulk-forming agents (e.g., psyllium) cause water absorption, bowel distention, and reflex bowel activity, with a 12-h to 3-d lag to onset of action. Osmotics (e.g., lactulose) increase the volume of liquid in the bowel lumen, with onset in 1-3 $\mathrm{h}$. Lubricants (e.g., liquid paraffin) coat the surface of the feces to aid passage, with a 6- to 8-h time to onset. Stimulants (e.g., bisacodyl) increase peristalsis via innervation, with an onset of 6-12 h; this category includes sodium picosulfate, typically used for bowel preparation for medical procedures. Combination therapy (e.g., softener and stimulant) can also be used.

Mode of Action. Bisacodyl is a stimulant laxative that increases water retention in the stool and stimulates peristalsis in the bowel $(5,6,10,18)$. As such, it is effective for clearing the bowel of fecal content but should not be used regularly for constipation $(5,6,10,18)$.

Pharmacokinetics. Bisacodyl has minimal absorption $(16,18)$. The small amount that may be absorbed after oral administration has $99 \%$ plasma protein binding, is metabolized in the liver, and is eliminated via the kidneys $(16,18)$. After oral administration, onset of action occurs within $6-8 \mathrm{~h}(16,18)$.

Usual Indications. The usual indications for bisacodyl are for preparation for medical procedures and for treatment of severe constipation $(5,10)$.

Use in Nuclear Medicine. Bisacodyl is used to differentiate interluminal bowel activity from pathologic accumulation 
by stimulating fecal progression or passage in studies in which colonic activity can obscure pathology (e.g., ${ }^{67} \mathrm{Ga}$-citrate studies for non-Hodgkin lymphoma or abdominal infection, and ${ }^{123}$ I-metaiodobenzylguanidine abdominal imaging).

Proper Use and Dose Administration. The oral dose of enteric coated tablets should not be taken within $1 \mathrm{~h}$ of ingestion of milk or antacids $(5,18)$. To avoid gastric irritation and abdominal cramping, the tablet should not be crushed or chewed (18). The oral dose is typically 5-15 $\mathrm{mg}$ as a single daily dose for constipation and up to 30 $\mathrm{mg}$ for bowel cleansing ahead of procedures.

Contraindications. Bisacodyl should not be used when the bowel is obstructed (10).

Warnings and Precautions. Because of liver metabolism of the small fraction absorbed, caution should be exercised in patients with liver impairment.

Adverse Reactions. There are few major adverse reactions other than the expected local reactions, which include gastric irritation and cramping $(10,18)$. Fluid and electrolyte depletion is possible $(16,18)$.

Common Interactions. Possible interactions with medications that change gastric acidity (e.g., $\mathrm{H}_{2}$ antagonists and proton pump inhibitors) may alter the effects of bisacodyl (16).

\section{Heparin}

General Information/Drug Class. There are 5 main categories of anticoagulant medications $(5,6,10,18)$ : vitamin $\mathrm{K}$ antagonists (e.g., warfarin), antithrombin III-dependent anticoagulants (e.g., fondaparinux), direct thrombin inhibitors (e.g., lepirudin), direct factor $\mathrm{X}$ inhibitors (e.g., rivaroxaban), and heparin and low-molecular-weight heparins.

Heparin is a substance comprising repeating units of a disaccharide attached to a central protein; as a result, this unfractionated form has a variety of molecular weights $(5,6,10,18)$. Heparin is formed in mast cells of the lung, liver, and intestinal mucosa $(5,6,10,18,19)$. Heparin used for human injection tends to be derived from either porcine mucosa or bovine lung $(5,18)$. This is an important consideration for patients with cultural beliefs that may prohibit the use of products originating from these animals. Fractionated heparins are the lower-molecular-weight heparins separated for more predictable use (actions and adverse reactions).

Mode of Action. Heparin combines with antithrombin III, which is a naturally occurring clotting factor found in plasma $(5,6,10,18,19)$. The heparin-antithrombin III complex has numerous actions $(5,6,10,18,19)$. It inactivates thrombin factor IIa (most pronounced effect) and factors IXa, Xa, XIa, and XIIa. Inactivation of thrombin prevents fibrin formation and activation of factors V and VIII, and inactivation of factor $\mathrm{Xa}$ prevents conversion of prothrombin to thrombin, which prevents the formation of fibrin from fibrinogen.

Heparin is useful in preventing clot formation and growth and in vivo venous thrombosis, but it does not break down existing clots.
Pharmacokinetics. Heparin is inactive orally and has an intravenous bioavailability that is somewhat erratic $(5,16)$. It is highly plasma protein-binding, and only a small fraction is excreted unchanged in urine $(16,18)$. The elimination is dose-dependent, with a rapid saturation process at higher doses and slower renal elimination at lower doses $(5,18)$. The elimination half-life, therefore, averages $1.5 \mathrm{~h}$ but ranges from $30 \mathrm{~min}$ for low doses to up to $6 \mathrm{~h}$ for higher doses $(5,16,18)$. After intravenous administration, onset of action occurs rapidly, with significant effects lasting less than 3-6 h $(5,16,18)$. Heparin does not cross the placenta $(5,18)$.

Usual Indications. Heparin is used to prevent and treat venous thromboembolism (without actually breaking it down) and to prevent blood clots during surgery (prophylactically) $(5,6,10,18,19)$. It is used intravenously in preference to oral warfarin because it has an immediate onset of action and can be reversed if necessary with protamine sulfate (18).

Use in Nuclear Medicine. Heparin is used in nuclear medicine to prevent blood clotting during blood labeling procedures or to maintain intravenous lines.

Proper Use and Dose Administration. The specific dose of heparin used will depend on the technique and local protocol. For example, some sites may use preheparinized blood tubes whereas others may add a small volume of heparin to a syringe containing blood. Caution should be exercised when considering the order of adding heparin because it can label to the radionuclide. A typical dose for blood labeling would be 10-15 units/mL of blood.

Contraindications. Heparin is contraindicated in patients with heparin hypersensitivity, acute bacterial endocarditis, and bleeding risk (e.g., recent surgery or childbirth, peptic ulcer, severe liver disease, severe hypertension, hemophilia, cerebral aneurysm, or hemorrhage) $(5,6,18,19)$.

Warnings and Precautions. Heparin should be used with caution when patients are concurrently using dextran, dipyridamole, or thrombolytics (18). It should also be used with caution in asthmatic patients, patients with an allergy to animal proteins, and patients with liver dysfunction $(18,19)$.

Adverse Reactions. Bleeding and hemorrhage are the most common adverse reactions to heparin $(5,10,18,19)$. Hypersensitivity reactions can occur $(5,10,19)$.

Common Interactions. Several important interactions can occur with heparin, including an increased risk of bleeding when used with other anticoagulants (e.g., aspirin and nonsteroidal antiinflammatory drugs), and this risk of hemorrhage is particularly concerning when heparin is used with thrombolytics, dextran, and dipyridamole (18).

\section{CONCLUSION}

Nuclear medicine technologists may encounter a wide variety of interventional or adjunctive medications-some in common use and others less so-in general nuclear medicine practice. An understanding of basic pharmacology for these 
drugs or class prototypes allows enhanced practice. Specifically, this deeper understanding of pharmacology, indications, contraindications, warnings, precautions, proper use, drug interactions, and adverse reactions for each medication helps to ensure that nuclear medicine technologists meet the minimum capabilities outlined in their scope of practice (2). This, in turn, translates to safer practice and better patient outcomes.

\section{DISCLOSURE}

No potential conflict of interest relevant to this article was reported.

\section{REFERENCES}

1. Currie G. Pharmacology, part 3A: interventional medications in renal and biliary imaging. J Nucl Med Technol. 2018;46:326-334.

2. SNMMI-TS Scope of Practice Task Force. Nuclear medicine technologist scope of practice and performance standards. J Nucl Med Technol. 2017;45: 53-64.

3. Currie G. Pharmacology, part 1: introduction to pharmacology and pharmacodynamics. J Nucl Med Technol. 2018;46:81-86D.

4. Currie G. Pharmacology, part 2: introduction to pharmacokinetics. J Nucl Med Technol. 2018;46:221-230.

5. Waller D, Renwick A, Hillier K. Medical Pharmacology and Therapeutics. 2nd ed. London, U.K.: Elsevier; 2006:211-455.

6. Katzung BG, Masters SB, Trevor AJ. Basic and Clinical Pharmacology. 12th ed. New York, NY: McGraw Hill; 2012:251-294.

7. Loveless VS. Drugs Used as Interventional Agents in Nuclear Medicine. Albuquerque, NM: University of New Mexico Health Services Center; 1998:9-10.
8. Yudd AP, Van Heertum RL, Masdeu JC. Interventions and functional brain imaging. Semin Nucl Med. 1991;21:153-158.

9. Leung DK, Heertum RL. Interventional nuclear brain imaging. Semin Nucl Med. 2009;39:195-203.

10. Rang H, Dale M, Ritter J, Flower R. Rang and Dale's Pharmacology. 6th ed. London, U.K.: Churchill Livingston; 2008:368-396.

11. Block JH, Beale JM. Wilson and Gisvold's Textbook of Organic Medicinal and Pharmaceutical Chemistry. 11th ed. Philadelphia, PA: Lippincott Williams \& Wilkins; 2004:601-617.

12. Vorstrup S, Henriksen L, Paulsen OB. Effect of acetazolamide on cerebral blood flow and cerebral metabolic rate for oxygen. J Clin Invest. 1984;74:1634-1639.

13. Vorstrup S, Brun B, Lassen NA. Evaluation of the cerebral vasodilatory capacity by the acetazolamide test before EC-IC bypass surgery in patients with occlusion of the internal carotid artery. Stroke. 1986;17:1291-1298.

14. Kiss B, Dallinger S, Findl O, Rainer G, Eichler H, Schmetterer B. Acetazolamide-induced cerebral and ocular vasodilation in humans is independent of nitric oxide. Am J Physiol. 1999;276:R1661-R1667.

15. Theobald T. Sampson's Textbook of Radiopharmacy. 4th ed. London, U.K.: Pharmaceutical Press; 2011:555-574.

16. Sweetman SC, ed. Martindale: The Complete Drug Reference. 26th ed. Chicago, IL: Pharmaceutical Press; 2009:561-1876.

17. Park H-M, Duncan K. Nonradioactive pharmaceuticals in nuclear medicine. J Nucl Med Technol. 1994;22:240-249.

18. Bryant B, Knights K, Salerno E. Pharmacology for Health Professionals. 2nd ed. Sydney, Australia: Mosby Elsevier; 2007:505-614.

19. Greenstein B. Rapid Revision in Clinical Pharmacology. New York, NY: Radcliffe Publishing; 2008:79-85.

20. Saremi F, Jadvar H, Siegel ME. Pharmacologic interventions in nuclear radiology: indications, imaging protocols, and clinical results. Radiographics. 2002;22:477-490.

21. Baxter K. Stockley's Drug Interactions 2011. Chicago, IL: Pharmaceutical Press; 2011.

22. Datz FL, Christian PE, Hutson WR, Moore JG, Morton KA. Physiological and pharmacological interventions in radionuclide imaging of the tubular gastrointestinal tract. Semin Nucl Med. 1991;21:140-152.

23. Mettler FA, Guiberteau MJ. Essentials of Nuclear Medicine Imaging. 6th ed. New York, NY: Elsevier; 2012:563-564. 